\title{
An Efficient multi-level Pre-processing Algorithm for the Enhancement of Dermoscopy images in Melanoma detection
}

\author{
D Jeba Derwin ${ }^{1}$, O JebaSingh ${ }^{2}$, B Priestly Shan ${ }^{3}$ \\ ${ }^{1}$ Assistant Professor, SRM-TRP Engineering College, Tiruchirapalli, Tamil Nadu, India, d.jebaderwin@gmail.com. \\ ${ }^{2}$ Associate professor, Arunachala college of Engineering for women, Kanniyakumari, Tamil Nadu, India, o.jebasingh@ rediffmail.com. \\ ${ }^{3}$ Professor, Chandigarh University, Mohali, Punjab, India, priestlyshan@gmail.com.
}

\begin{abstract}
In this paper, a multi-level algorithm for Pre-processing of dermoscopy images is proposed, which helps in improving the quality of the raw images, making it suitable for skin lesion detection. This multi-level pre-processing method has a positive impact on automated skin lesion segmentation using Regularized Extreme Learning Machine. Raw images are subjected to de-noising, illumination correction, contrast enhancement, sharpening, reflection removal and virtual shaving before the skin lesion segmentation. The NLM filter with lowest BRISQUE score exhibits better de-noising of dermoscopy images. To suppress uneven illumination, gamma correction is subjected to the de-noised image. RICE algorithm is used for contrast enhancement, produces enhanced images with better structural preservation and negligible loss of information. Unsharp Masking for sharpening exhibits low BRISQUE scores for better sharpening of fine details in an image. Output images produced by the phase-congruency based method in virtual shaving shows high similarity with groundtruth images as the hair is removed completely from the input images. Obtained scores at each stage of pre-processing framework shows that, the performance is superior compared to all the existing methods, both qualitatively and quantitatively, in terms of uniform contrast, preservation of information content, removal of undesired information and elimination of artifacts in melanoma images. Output of proposed system is assessed qualitatively and quantitatively with and without pre-processing of dermoscopy images. From the overall evaluation results it is found that, the segmentation of skin lesion is more efficient using Regularized Extreme Learning Machine if the multi-level pre-processing steps are used in proper sequence.
\end{abstract}

Keywords: Non-Local Means Filter, Robust Image Contrast Enhancement, Unsharp masking, Dermoscopy, Phase congruency

\section{INTRODUCTION}

Melanoma is the most common deadliest skin cancer, with 91,000 new cases annually in US and causes more than 9000 deaths [1]. Globally, skin cancer is one of the life-threatening disease in western countries. In Europe, more than 100,000 new melanoma cases, with 22,000 deaths are reported yearly [2]. The statistics are all more alarming that, unlike other types of cancer melanoma has been steadily increasing over the past decades. Consequently, early detection of melanoma is a significant challenge in the diagnosis and treatment of skin cancer. Over recent years, a high-resolution dermoscopy skin imaging technique is used to visualize the deep skin structures. Although, dermoscopy images are of 
high-resolution the visualization of images is still subjective due to poor contrast, skin tone variations, non-uniform illumination and artifacts [3] . A small amount of noise is present in the dermoscopy images may get amplified during sharpening and contrast enhancement. The amplified noise may adversely affect the performance of edge-based segmentation algorithms used to extract the borders of the skin lesions. Hence, de-noising is a vital step in the automated analysis of dermoscopy images.

Mostly, skin lesions are darker than the background. However, due to uneven illumination, some portions of the image may appear darker than the background. Those darker regions may get falsely segmented along with the lesions. Therefore, contrast enhancement and sharpening are indispensable in the automated analysis of dermoscopy images. Specular reflection is another concern that may deteriorate the visual quality of melanoma images. Hence, reflection removal is needed to eliminate the background reflections in input images. Hairs are present in dermoscopy images. The hairs being dark, they may get falsely segmented along with the lesion, if intensity-based segmentation methods are adopted. Hairs need to be removed prior to the segmentation of lesions. The process of removing hairs from dermoscopy images is usually termed as virtual shaving.

In this paper, a new six-stage pre-processing algorithm is introduced to improve the segmentation accuracy of skin lesion in dermoscopy images. For de-noising the input image, the Non-Local Means (NLM) filter is employed. It ensures the preserving of detail information of an image. Likewise, gamma correction is applied at the second stage so that a uniform illumination is achieved. An algorithm termed as Robust Image Contrast Enhancement (RICE) is employed for contrast enhancement. This method helps in avoiding the over contrast enhancement. For sharpening, Unsharp Masking technique is implied to sharpen the edge pixels. For reflection removal, a transmittance estimation-based strategy is adopted. As a result, the undesired information is removed thereby improving the visual quality. Under virtual shaving, a phase congruency-based method is adopted for removing the hairs without losing the image content. The implemented technique in each stage performs efficiently such that a quality image is achieved at the pre-processed output for melanoma segmentation. The output of proposed system is evaluated subjectively with ground truth images and objectively using quality metrics like Disc Similarity Index (DSI), Jacquard Index (JI) and Total Segmentation Coefficient (TSC). The output results reveal, the multi-level pre-processing algorithm outperforms in the segmentation of skin lesion using Regularized Extreme Learning Machine (RELM).

\section{LITERATURE SURVEY}


To enhance the dermoscopy image, Madhan kumar et al.[4]presented a preprocessing technique in two-steps to remove the noise, fine hairs and air bubbles. Accordingly, the contrast of an input image is enhanced by Histogram Equalization and the reduction of impulsive noise, hair structures and air bubbles is achieved by applying the median filter. Although it preserves the edges, the fine image details are lost when the window size of the filter is increased above 3X3. Furthermore, Jaworek et al. [5] proposed a novel method to reduce the border irregularity in dermoscopy images. The authors highlighted a two-step pre-processing algorithm includes Black frame removal, Hair detection and In painting. Initially, each row of an image is scanned in four directions and the rows with $50 \%$ of black pixel are removed in the input image. Next, the Black top-hat transform is applied to remove the dark thick hairs from black frame removal image. Here, the Black top-hat transform has failed to detect the local structures such as dots or globules in Melanoma images. Moreover, Restrepo et al. [6] introduced a contrast enhancement technique based on the most discriminant projection of the color map in skin lesion images. This method overcomes the non-uniform illumination and color correction problems while detecting the Melanoma. Since, the color projection is calculated for all directions, it increases the complexity of the algorithm. In addition, a five-step pre-processing framework is proposed by Mishraet al. [7] includes: Elimination of lighting effects, color correction, contrast enhancement, image smoothing and hair removal to improve the visual quality of the image. Here, the authors highlighted the problems in skin lesion detection like poor contrast, skin tone variation, artifacts and non-uniform illumination on dermoscopy images.

Furthermore, Cherepkova et al. [8] proposed an enhancement and color correction for original dermoscopy images. In this article, enhancement is achieved in sixsteps including: Retinex, Spatio Temporal Retinex-inspired Envelope with stochastic sampling, Automatic White Balance (AWB), contrast enhancement, automatic enhancement and histogram equalization. The authors reported an improved sensitivity and accuracy with an average of 4 to $8 \%$ and 3 to $5 \%$ respectively. Due to over exposure in visual adjustment, fine image details are lost with partly corrected color. Although, AWB provides a good color correction, some deviations in visual quality occurs due to the errors in temperature estimation. Also, a two-phase pre-processing algorithm for dermoscopy image enhancement is proposed by Jayalakshmi etal. [9]. Accordingly, a median filter is applied to remove the artifact and K-means clustering is used to eliminate the outlier pixels. The presented result shows an accuracy of $92.8 \%$ with sensitivity of $93 \%$ and specificity of $90 \%$ on Danderm database.

Furthermore, a three-step framework was proposed to improve the contrast of the dermoscopy images in [10]. Initially, a Median filter is employed to reduce noise in the 
raw input images. Next, the morphological operators such as erosion and dilation are implemented to remove the artifacts like hairs in the filtered image. Finally, intensity value mapping is applied to enhance the contrast. Through median filtering, a $5 \times 5$ window is used to remove the image details of 2 pixel wide. Pankaj at al. [11] introduced a reformed contrast enhancement technique using Krill Herd $(\mathrm{KH})$ optimization. Here, a new reformed histogram is obtained with peaks cut off. The global histogram equalization helps in the enhancement of medical images like X-ray, MRI and CT scan. In this approach, the efficiency is tested through the metrices like SSIM, EPI, DE and REC. Jeevakala et al. [12] discussed a sharpening enhancement technique for MR images. Laplacian Pyramid and singular value decomposition is implemented to decompose the multi-scale images into coarse and difference sub-bands. Here, the weighted sum of singular matrix and its Global Histogram Equalization increases the contrast in multi-scale images.

Though lot of literatures are enumerated in pre-processing of dermoscopy images, some limitations are identified as follows:

$>$ Normally, Median filters are used for de-noising in dermoscopy images. In such methods, when the filter size is increased above $3 \times 3$, fine details of the image are lost.

$>$ The black-hat transform implemented for hair removal is unable to remove local structures like dots and globules.

$>$ Automatic White Balance (AWB) causes over exposure in visual adjustment, leads to loss of fine image content.

$>$ The over enhancement and multiple illumination artifacts are found in Contrast Limited Adaptive Histogram Equalization (CLAHE), Contextual and Variational Contrast enhancement algorithm (CVC) and Layered Difference Representation (LDR) algorithms.

$>$ Moreover, in existing methods the hairs are removed using median filters leads to loss of image information.

Inorder to overcome the above issues and enhance the spatial quality for skin lesion segmentation in dermoscopy images a pre-processing module comprising of de-noising, illumination correction, contrast enhancement, sharpening, reflection removal and hair removal are introduced in this work. Under de-noising phase, the NLM filter with suitable DoS value is chosen to preserve the fine details of dermoscopic images. Also, in the contrast enhancement phase, the RICE algorithm is introduced to avoid the non-uniform enhancement by maintaining a mean brightness. In addition, the reflection removal is proposed to remove undesired information by separating the background image layer from the reflection layer of the dermoscopy image to be analyzed. Thus, by optimizing the SP and 
RCP values in reflection removal process the visual quality of the image is also preserved. Moreover, a phase congruency method with ideal threshold value preserves the image content in virtual shaving of hairs. The rest of the paper is organized as follows: Section III explains the pipeline of dermoscopy pre-processing method in detail. Section IV describes the results and discussion. Finally, section $\mathrm{V}$ draws the conclusion.

\section{METHODOLOGY}

In this paper, a pre-processing methodology is introduced for dermoscopy images which can improve the visual quality of digital images in order to achieve an accurate segmentation. The schematic representation of the flow of work is depicted in Fig. 1.

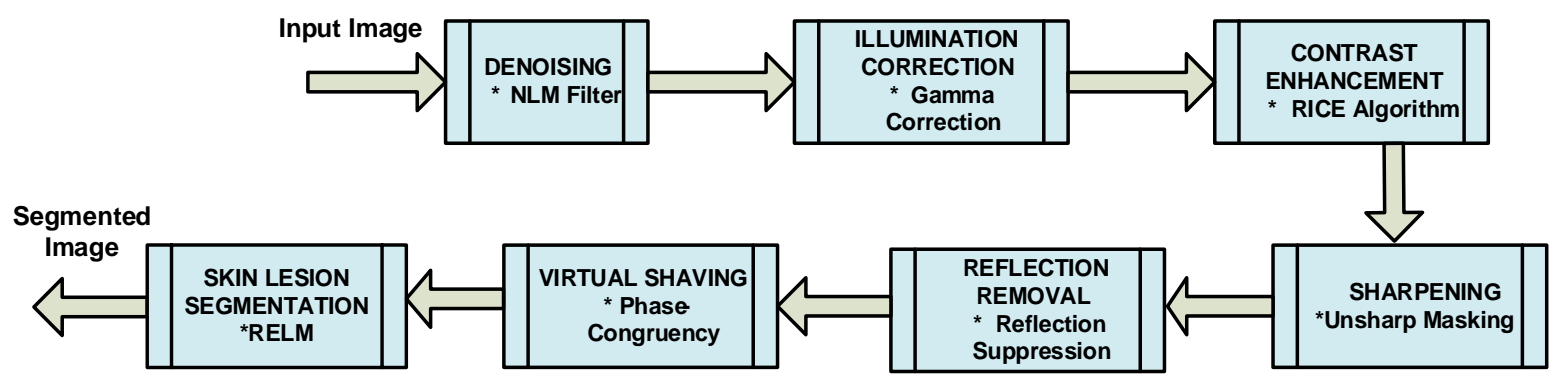

Fig. 1 Schematic representation of flow of work

\subsection{De-noising:}

In the proposed method, NLM filter is used to perform the objective of de-noising [13]. Therefore, for estimating the denoised pixel value $Y(m, n)$ of an input image pixel $X(m, n)$, a windowing technique is applied on each $3 \times 3$ block of input dermoscopy images. Hence, $\mathrm{Y}(m, n)$ is computed as the weighted sum of the pixel values inside a block with radius $R_{1}$ as:

$$
Y(m, n)=\sum_{i=-R_{1}}^{+R_{1}} \sum_{j=-R_{1}}^{+R_{1}} W[X(m, n), X(m+i, n+j)] X(m+i, n+j),\left\{\begin{array}{l}
1 \leq m \leq M \\
1 \leq n \leq N
\end{array}\right\}
$$

where, $\mathrm{M}$ and $\mathrm{N}$ indicates the number of rows and columns in the input image. The weights $W(m, n)$ are based on the similarity of neighborhood pixels $m$ and $n$. The similarity is then estimated as:

$$
W[X(m, n), X(m+i, n+j)]=e^{\frac{-\left[\sum_{p=-R_{2}}^{+R_{2}} h_{g}[X(m+p, n+p)-X((m+i)+p,(n+j)+p)]^{2}\right]}{\xi^{2}}}
$$


the variable, ' $h_{g}$ ' is a normalizing constant. It penalizes the grey level difference of the pixels within the similarity-block, which are away from its center. Now equation (2) is subjected to a normalization process,

$$
\begin{aligned}
& 0 \leq W[X(m, n), X(m+i, n+j)] \leq 1 \& \\
& \sum_{i=-R_{1}}^{+R_{1}} \sum_{j=-R_{1}}^{+R_{1}} W[X(m, n), X(m+i, n+j)]=1
\end{aligned}
$$

After normalization of the weights, the weight corresponding to the pixels, which are closely similar to the pixel to be denoised will get penalized more. Towards rectifying this inadvertent problem, the weight corresponding to the self-similarity is replaced by the highest value of weight just below it. Therefore, the weight $W X(m, n), X(m+i, n+j)]$ at $i=0$ and $j=0$ is expressed as:

$$
\begin{aligned}
& \max (W[X(m, n), X(m+i, n+j)]) \forall i \neq 0 \& \\
& \qquad j \neq 0,-R_{1} \leq i \leq+R_{1},-R_{1} \leq j \leq+R_{1}(4)
\end{aligned}
$$

The variable, ' $\xi$ ' is an arbitrarily-defined operational parameter of the NLM filter, called as 'decay control parameter'. It is otherwise called as 'Degree of Smoothing (DoS). To

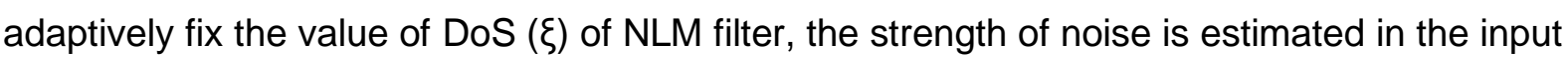
image. In this paper, the value of DoS is linearly proportional to the Standard Deviation (SD) of noise in the input image. This can be done as:

$$
\xi=\beta \widehat{\sigma}_{n}
$$

where, ' $\widehat{\sigma}_{n}$ ' indicates the SD of zero mean additive Gaussian noise.

\subsection{Illumination Correction:}

To suppress the uneven illumination in the denoised image $Y$, illumination correction is implemented in the dermoscopy images. Hence, to suppress the uneven illumination, gamma correction is subjected to the illumination component of HSV color space. Initially, the denoised input image in RGB color space is converted to the HSV color space. Here, the hue component and saturation component are kept intact and the value component alone is decomposed using Retinex decomposition. Later, the estimated illumination component is subjected to the Gamma correction to suppress the unevenness. Since, the arbitrary parameter ' $r$ ' controls the effectiveness of the devignetting called as Devignetting Quality Parameter (DQP). In this work, the DQP value is varied between 0.25 and 2.5 and the best value is selected as 2.0. Then, the new value component is reconstructed from the decomposed reflectance component and gamma corrected illumination component. Finally, 
combining the hue, saturation and new value components together an illumination corrected image $Y_{i}$, is obtained by converting the resultant image in HSV color space to RGB color space.

\subsection{Contrast Enhancement:}

To increase the gray level difference between the lesion and background of an illumination corrected image $Y_{i}$, the RICE algorithm is implemented in dermoscopy images.

Initially, the histogram $h_{i}$ and Equalized histogram $h_{e q}$ is obtained for the input image. Later, by applying sigmoid transfer mapping function $T_{s i g}($.$) , the corresponding histogram$ $h_{\text {sig }}$ is obtained which improves the visual quality of the image. Now, the target histogram $\tilde{h}$ is estimated as:

$$
\tilde{h}=\frac{h_{i}+\Phi h_{e q}+\psi h_{s i g}}{1+\Phi+\psi}
$$

Where $\Phi$ and $\psi$ are the control parameters, selected based on the saliency preservation. It is measured by a Quality assessment Metric of Contrast (QMC) [14] in an image. Finally, the contrast enhanced image, $Y_{c}$ can be reconstructed using histogram matching function $T_{h m}().[15]$.

$$
Y_{c}=T_{h m}\left(Y_{i} \tilde{h}(\Phi, \psi)\right)
$$

\subsection{Sharpening:}

The principle of Unsharp Masking is exclusively based on the concept of estimating difference between the input image and the Gaussian filtered image [16]. A fraction of the high-frequency content is computed by subtracting the Gaussian filtered image from the input image. Again it is added back to the input image to get the unsharp masking.

To perform the unsharp masking, the Gaussian filter kernel is used to compute Gaussian filter mask $H_{G}$ is given by,

$$
H_{G}(x, y)=\frac{1}{2 \pi \sigma^{2}} e^{-\left(\frac{x^{2}+y^{2}}{2 \sigma^{2}}\right)},-w \leq x \leq+w \text { and }-w \leq y \leq+w
$$

Selecting the dimension of Gaussian mask and its SD is important to make the strength of smoothing more sensitive. Therefore, SD is computed from the value of the radius of the mask. The SD of Gaussian mask from its radius is computed using the relation $\sigma=(w-1) / 4$. According to this relation, when the radius of the Gaussian masks increases the SD also increases proportionally. Therefore, when both SD and dimension of the mask 
increases together, the degree of smoothing also increases significantly. The identity convolution mask, ' $\mathrm{H}_{0}$ ' can be calculated as:

$$
H_{0}(x, y)=\left\{\begin{array}{cc}
1 & x=0 \& y=0 \\
0 & \text { Otherwise }
\end{array}-w \leq x \leq+w \text { and }-w \leq y \leq+w\right.
$$

Finally, the sharpened image $Y_{S}$ is obtained by computing the difference between the input image $Y_{c}$ and its Gaussian filtered output.

$$
Y_{S}=Y_{C} * * H_{0}+\lambda\left(\left[H_{0}-H_{G}\right] * * Y_{c}\right) \quad 0 \leq \lambda \leq 1
$$

The fraction of difference between the input and the Gaussian filtered image merged to the input image is a manually selected parameter $\lambda$. This parameter is usually called as scale and if the value of $\lambda$ is more the sharper will be the output image.

\subsection{Reflection removal}

It is important to remove the undesired reflections, the reflection removal is implemented in the sharpened image. The process of reflection suppression is based on enhancing the image quality by separating the reflectance layer from the transmittance layer [17]. Based on this observation, an RGB image can be represented as the weighted sum of its transmittance layer and reflectance layer as explained in (12).

$$
Y_{s}=\Gamma(W, T)+\Gamma(1-W, k * * R)
$$

where, $Y_{S}$ is the input RGB image. The variable, ' $T$ ' indicates the transmittance layer and the variable ' $R$ ' indicates the reflectance layer of the input image. The notion ' $\Gamma$ ' indicates element-wise multiplication. The notion, ' $* *$ ' denotes the $2 \mathrm{D}$-convolution operation. ' $W$ ' indicates the matrix that weighs the contribution of the transmittance layer at each pixel. ' $k$ ' is the blurring kernel. The weighing matrix, ' $W$ ' is expressed as:

$$
W_{m, n}=w, \forall m, n, \quad 1 \leq m \leq M, 1 \leq n \leq N
$$

To avoid losing the high frequency component during reflectance removal the Laplacian based data fidelity is taken in the sharpened image. The optimization problem developed for reflection removal image $Y_{r}$ is described as:

$$
Y_{r}=\underset{T}{\operatorname{argmin}}|| \mathcal{L}(T)-\left.\mathcal{L}\left(Y_{S}\right)\right|_{2} ^{2}+\lambda C(T)
$$

where, $\lambda$ is the regularization parameter and if $\lambda$ value increases more gradients will be removed. The term $C(T)$ invigorates the smoothening of image without disturbing the continuity of large structures. 


\subsection{Virtual Shaving:}

The process of removing hairs from dermoscopy images is usually termed as virtual shaving. The hairs being dark, they may get falsely segmented along with the lesion. Phase Congruency based virtual shaving method is adopted for the removal of hairs. In the first step of hair removal, the color image is converted to grayscale.

Hairs are detected from the grayscale image based on its phase congruency. A 2DLog Gabor Filter (LGF) is used for computing phase congruency of the image [18].The final phase congruency model of the image is given by:

$$
\phi(m, n)=\frac{\sum_{s} \sum_{o} w_{o}(m, n)\left\lfloor A_{s o}(m, n) \Delta \phi_{s o}(m, n)-T\right\rfloor}{\sum_{s} \sum_{o} A_{s o}(m, n)+\xi_{s}}, 1 \leq m \leq M, 1 \leq n \leq N
$$

where, $T$ is the noise-compensation term, $w_{o}$ represent a weighting function, $\Delta \phi_{s o}$ term represents a phase deviation function and the variable, $\xi_{s}$ is a minute value used to avoid computational indeterminacy.

By applying threshold on phase congruency model of an image the phase angle $\Phi_{P}$ is estimated as:

$$
\Phi_{P}(m, n)=\left\{\begin{array}{ll}
1, \text { if } \phi(m, n)<0 \\
0, & \text { otherwise }
\end{array}, 1 \leq m \leq M, 1 \leq n \leq N\right.
$$

The modified phase angles $\Phi_{N 1}$ is theresult of negative phase angles modified in the range 0 to $\pi$ is expressed as:

$\Phi_{N 1}(m, n)=\Phi_{P}(m, n)(-\phi(m, n))+\Phi_{P}^{\prime}(m, n) \phi(m, n), \quad 1 \leq m \leq M, 1 \leq n \leq N$

where, the variable $\Phi_{P}^{\prime}$ is the complement of $\Phi_{P}$. Again the phase angles in $\Phi_{N 1}$ are modified such that, the angles greater than $\frac{\pi}{2}$ are brought to 0 to $\frac{\pi}{2}$ is given by:

$\Phi_{N 2}(m, n)=\Phi_{P 2}(m, n)\left(\pi-\Phi_{N 1}(m, n)\right)+\Phi_{P 2}^{\prime}(m, n) \Phi_{N 1}(m, n), 1 \leq m \leq M, 1 \leq n \leq N$

The term $\Phi_{P 2}$ indicate the locations where $\Phi_{N 1}$ is greater than $\frac{\pi}{2}$ and variable $\Phi_{P 2}^{\prime}$ is the complement of $\Phi_{P 2}$.

The modified phase angles are then normalized as:

$$
\Phi_{R}(m, n)=\frac{\frac{\pi}{2}-\Phi_{N 2}(m, n)}{\frac{\pi}{2}}, 1 \leq m \leq M, 1 \leq n \leq N
$$


Later, the phase values ' $\Phi_{R}$ ' are converted to binary with a threshold, ' $t$ '.

$$
\Phi_{b}(m, n)=\left\{\begin{array}{l}
1, \text { if } \Phi_{R}(m, n)<t \\
0, \quad \text { otherwise }
\end{array}, 1 \leq m \leq M, 1 \leq n \leq N\right.
$$

Now the binary phase image $\Phi_{b}$ is then dilated with disk-shaped structural element $S E$. The dilation in the binary image makes the objects visible by filling the small holes in it. Hence, the dilated phase image $\Phi_{D}$ is given by:

$$
\Phi_{D}=\Phi_{b} \oplus S E
$$

where, $S E$ is the structural element described as: $S E=\left[\begin{array}{lll}0 & 1 & 0 \\ 1 & 1 & 1 \\ 0 & 1 & 0\end{array}\right]$

Then, the connected components $P$ are found on the dilated binary phase image $\Phi_{D}$. Eccentricity is calculated for each of the connected regions. Hairs like structures are elliptical structures with eccentricity close to 1 .

$$
H_{i}=\left\{\begin{array}{c}
1, \text { if } E_{i}<t_{b} \\
0, \text { otherwise }
\end{array}, \quad 1 \leq i \leq P\right.
$$

The region without hairs are indicated as $H_{i}$ and the threshold ' $t_{b}$ ' is arbitrarily selected as 0.6. The resulted virtual shaving image $Y_{v}$ for RGB channel without hairs after region filling is given by:

$$
Y_{v R}=\Psi\left(Y_{r R}, H_{i}\right), Y_{v G}=\Psi\left(Y_{r G}, H_{i}\right) a n d Y_{v B}=\Psi\left(Y_{r B}, H_{i}\right), 1 \leq i \leq P
$$

where, $\Psi$ ' indicates the region filling operator and $Y_{r}$ is the reflection removed image.

\subsection{Segmentation}

Lesion segmentation means separating the lesion region from the normal skin region. It is a crucial step in the analysis of dermoscopy images to identify various global morphological features of the lesion. RELM with ridge regression is employed for segmentation of skin lesion in the proposed system. Based on the ridge regression model, the stable and better regularization can be achieved by adding $1 / C$ to the diagonal elements $P^{\top} P$ while estimating the output weight ' $\beta$ '.

Thus, the RELM regression becomes

$$
P^{+}=\left(P^{\top} P+I / C\right)^{-1} P^{\top}
$$

where ' $\mathrm{l}$ ' is an identity matrix

Based on the matrix inversion property (25) can be written as 


$$
P^{+}=P^{\top}\left(P P^{\top}+l / C\right)^{-1}
$$

In order to reduce the computation power, (25) \& (26) can be selected based on $\mathrm{P}^{\top} \mathrm{P}$ or $\mathrm{PP}^{\top}$ with smaller dimensions. Therefore, the computation complexity of RELM can be estimated as follows:

$$
\beta=\left[P^{\top} P+l / C\right] P^{\top} T
$$

where ' $T$ ' stands for target estimation and ' $\mathrm{P}$ ' is the hidden neuron matrix Also, (25) \&(26) aims at optimizing $\|P \beta-T\|^{2}+1 / C\|\beta\|^{2}$ shows that smaller output weights $\beta$ plays a vital role in better generalization of RELM. The procedure of RELM is given in three steps

Step 1: - Randomly estimate the hidden neuron parameters, weight ' $w$ ' and bias 'b'

Step 2: - Estimate the hidden layer matrix 'P' using

$$
\mathrm{P}=\left[\begin{array}{c}
P_{1} \\
\vdots \\
P_{N}
\end{array}\right]=\left[\begin{array}{c}
P\left(x_{1}\right) \\
\vdots \\
P\left(x_{N}\right)
\end{array}\right]=\left[\begin{array}{ccc}
G\left(w_{1}, b_{1}, x_{1}\right) & \cdots & G\left(w_{L}, b_{L}, x_{L}\right) \\
\vdots & \ddots & \vdots \\
G\left(w_{1}, b_{1}, x_{N}\right) & \cdots & G\left(w_{L}, b_{L}, x_{N}\right)
\end{array}\right]
$$

Step 3: - Calculate the output weight ' $\beta$ ' using

$$
\beta=H^{+} \mathrm{T}
$$

where $\mathrm{H}^{+}$derived from (25) \& (26)

Since hidden neuron parameters are randomly chosen, fast learn speed is achieved in RELM. Due to randomness nature, ELM and other ANN algorithms have high variance and prediction error. In this case, ridge regression is quite beneficial in the reduction of variance and prediction error due to smaller value of output weight ' $\beta$ '. Also, the over fitting problem is addressed with regularization parameter ' $C$ ' in RELM produces better and consistent performance than other segmentation algorithms.

\section{RESULTS AND DISCUSSION}

The quality of proposed system is analyzed subjectively and objectively in this section. Twelve objective quality metrics are used in this section. They are (1) Blind Reference less Image Spatial Quality Evaluator (BRISQUE) (2) Average Gradient of the Illumination Component (AGIC) (3) Lightness Order Error (LOE) (4) Sparse Feature Fidelity (SFF) (5) Visual Saliency-based Index (VSI) (6) Patch-based Contrast Quality Index (PCQI) (7) Over-Contrast measure (OCM) (8) Cumulative Probability of Blur Detection (CPBD) (9) 
Smoothing Parameter (SP) (10) Rate Control Parameter (RCP) (11) Peak Signal to Noise Ratio (PSNR) and (12) Structural Similarity Index Metric (SSIM).

\subsection{Image Dataset:}

The dermoscopy images are collected from the data archive of International Skin Imaging Collaboration (ISIC) [18]. The archive comprises a total of 900 dermoscopy images. The test data of the ISIC Melanoma Challenge 2016 is used in our experiment. The data comprises of 379 images. Out of 379 images, 273 images comprises melanoma. A total of 106 images are of normal lesions. Images with malignant lesions are labeled after performing the biopsy. All images comprising benign lesions are labeled after a histopathological examination and prolonged longitudinal follow up. Associated ground-truth segmentation contoured by the expert dermatologists are also provided in the archive.

\subsection{Validation of NLM filter}

The influence of DoS on the de-noising quality of NLM filter is analyzed subjectively and objectively in this section. Under objective evaluation, the BRISQUE score is evaluated. The test images are filtered by NLM filter, by varying DoS values from 1 to 15 and the results of some DoS values are shown in Fig. 2. As the value of the DoS varies for 1 to 15 , the smoothing effect on the images also increases. It is evident from fig. 2 (b-f), when the value of DoS increases beyond ten, the images become excessively smoothed. This weakens the lesions present in the images. Hence, based on the perceived quality of processed images, the range of DoS between 6 and 9 is observed to be suitable for dermoscopy images.

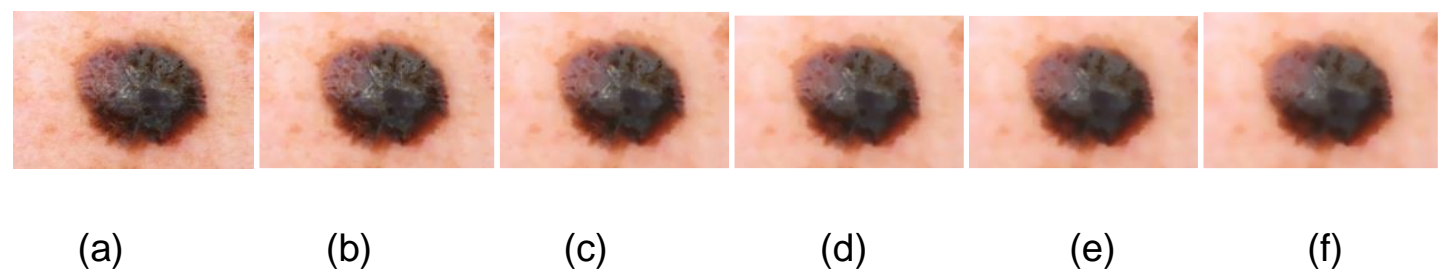

Fig. 2 Output images produced by NLM filter for different values of DoS (a) Test image (b) DoS = 3 (c) DoS = 5 (d) DoS = 7 (e) DoS = 9 (f) DoS $=11$

The variations of BRISQUE score for different values of DoS plotted for three test images are depicted in Fig. 3. In this graph, it shows a low BRISQUE score when DoS is varied between 8 and 10. As the DoS increases beyond 10, the BRISQUE score also increases for all the three images. 


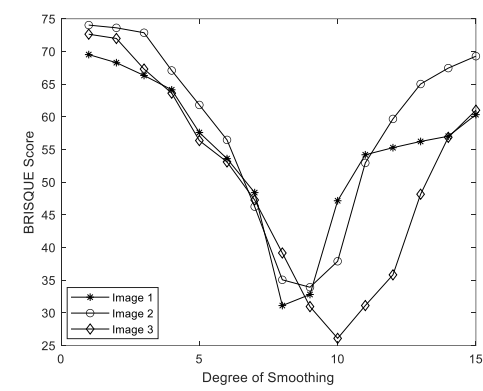

Fig. 3 Variation of BRISQUE score against DoS.

NLM filter is compared qualitatively and quantitatively against two different alternatives of de-noising namely, Anisotropic Diffusion Filter (ADF)[19] and Bilateral Filter (BF)[20]. In Fig. 4(c) BF excessively smoothens the image that greatly reduces the sharpness of the denoised image and thereby fades the boundary of the lesions. Likewise, in Fig. 4(b) the image denoised by ADF shows the boundary of lesions are not preserved properly with textural artifact. But in Fig. 4(d), the image is properly denoised by NLM filter by maintaining the boundary of the lesions than the ADF. The Information loss is also minimal when compared to the bilateral filter.

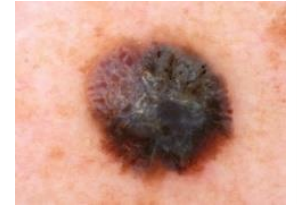

(a)

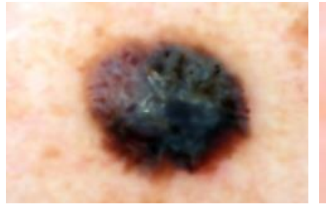

(b)

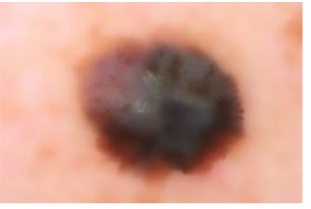

(c)

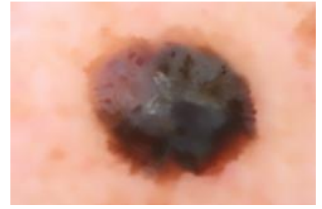

(d)

Fig. 4: Output images produced by different de-noising algorithms (a) test image (b) ADF (c) BF (d) NLM.

The summary of BRISQUE scores obtained for ADF, BF and NLM for 100 images is tabulated in Table 1. It is evident that the NLM filter obtains the least value of BRISQUE score compared to the other schemes.

Table 1 BRISQUE scores shown by various de-noising schemes

\begin{tabular}{ccccc}
\hline Method & Image 1 & Image 2 & Image 3 & $\begin{array}{c}\text { Summary on 100 } \\
\text { images }\end{array}$ \\
\hline ADF & 48.4356 & 53.7685 & 52.5846 & $51.0745 \pm 3.4252$ \\
BF & 40.6547 & 42.3425 & 43.5926 & $42.2393 \pm 4.3343$ \\
NLM filter & 31.2365 & 34.5476 & 33.4826 & $33.1646 \pm 2.3256$ \\
\hline
\end{tabular}




\subsection{Validation of illumination correction}

The influence of DQP on the quality of devignetted images is analyzed subjectively and objectively. For objective analysis, the quality metrics like AGIC, LOE, SFF, and VSI are used. Based on this analysis, identifying the suitable range of DQP for illumination correction in dermatological photographs is important. The output images corresponding to the proposed devignetting scheme for different values of DQP are depicted in Fig. 5. It is observed that in Fig. 5(b), when the value of the DQP is less than one, the grey levels at the enhanced regions in the input images get compressed or scaled-down. In effect, the dynamic range of the processed images gets compressed and it appears to be relatively darker than the input images. If the value of the DQP is equal to one, the processed image becomes exactly similar to the corresponding input images as shown in Fig. 5(c).

When the value of $D Q P$ is above one $(D Q P=1.5)$, the darker regions of the input images become enhanced slowly and the background illumination becomes uniform. However, the vignetting error is not fully corrected, it can be seen in Fig. 5(d). For the value, $(\mathrm{DQP}=2)$ the dark corners of the dermatological photographs caused by the vignetting error become equally enhanced as the bright regions in the photographs is depicted in Fig. 5(e). In case, if DQP is greater than $2(D Q P=2.5)$ an over-enhancement can be noticed in Fig. $5(f)$. Therefore, $D Q P=2$ is chosen as the optimized value for illumination correction due to uniform brightness throughout the image.

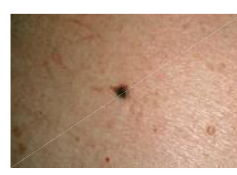

(a)

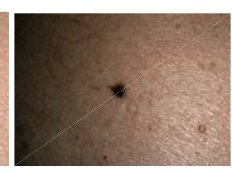

(b)

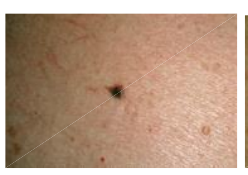

(c)

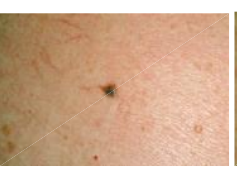

(d)

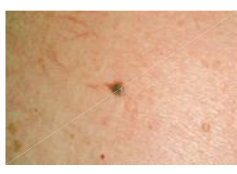

( e )

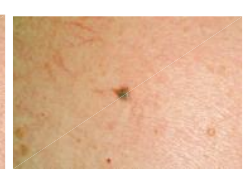

(f)

Fig. 5 Outputs of the proposed devignetting scheme (a) Test image (b) DQP $=0.5$

(c) $\mathrm{DQP}=1$

(d) $\mathrm{DQP}=1.5$

(e) $D Q P=2$ (f) DQP $=2.5$

The variations of AGIC, LOE, SFF and VSI with respect to DQP are shown in Fig. 6(a-d). In Fig. 6(a), the AGIC monotonically decreases as the DQP increases. AGIC becomes almost consistent for the values of DQP greater than 2. In Fig. 6(b), the LOE continuously decreases when the value of $\mathrm{DQP}<1$ and reaches minimum at a point where $D Q P=1$. Afterwards, the LOE increases linearly when the value of DQP is greater than 1. In Fig. 6(c) and 6(d), when DQP changes from 0 to 1, both SFF and VSI increases and reaches the maximum point at $D Q P=1$. When $D Q P$ increases above 1 , the SFF and VSI start decreasing and above 2.2 the slope of SFF and VSI increases. This analysis of AGIC, LOE, SFF and VSI with respect to DQP indicates the optimum value of DQP suitable for the dermatological images. 


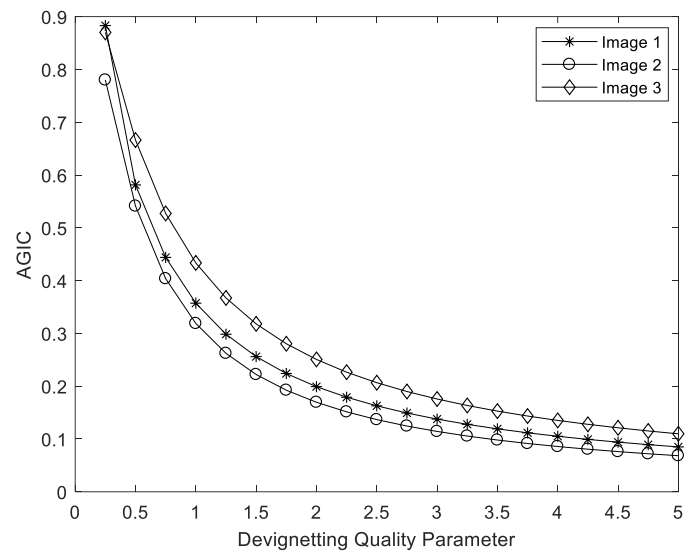

(a)

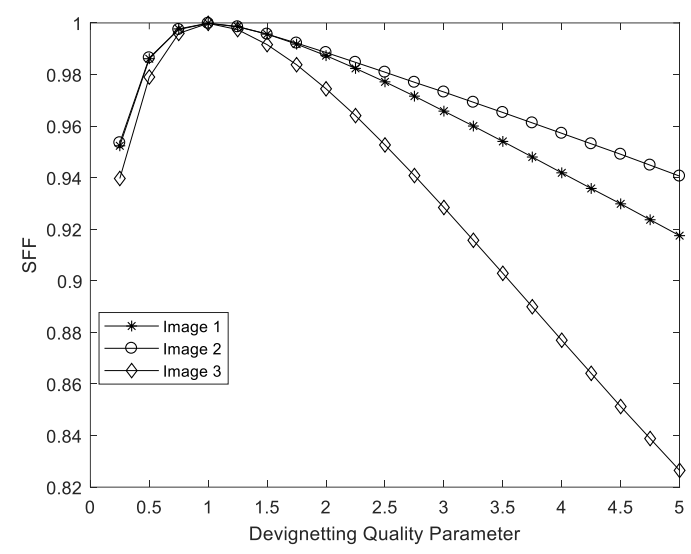

(c)

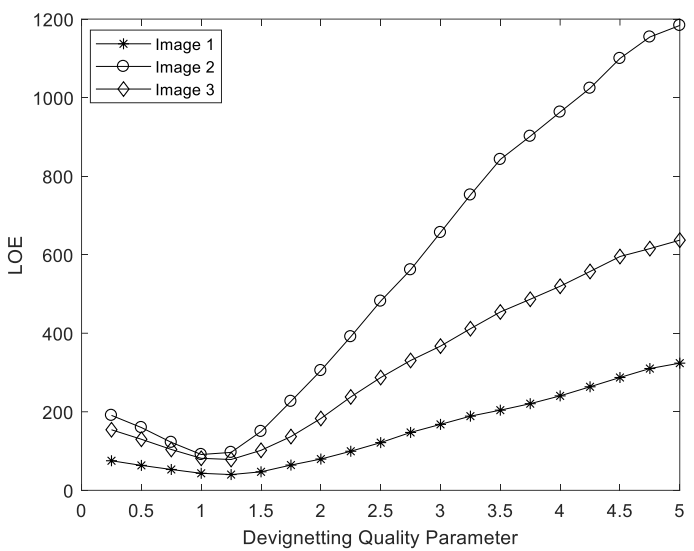

(b)

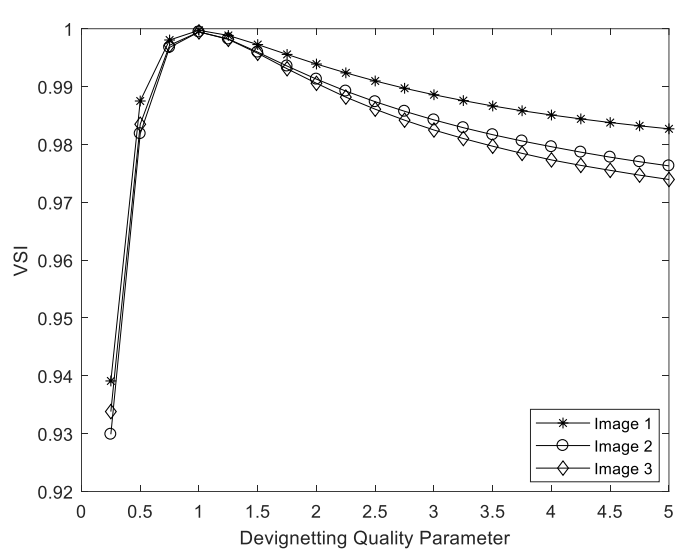

(d)

Fig. 6: Variation of the objective quality metrics with respect to DQP (a) AGIC Vs DQP (b) LOE Vs DQP (c) SFF Vs DQP (d) VSI Vs DQP

The proposed devignetting algorithm is compared both qualitatively and objectively, against three different algorithms namely, Gamma Correction (GC)[21], Variation-based Fusion (VF)[22] and Sigmoid Transform (ST)[23]. The obtained images by applying different devignetting algorithms are depicted in Fig. 7. An ideal devignetting technique should make the background illumination uniform throughout the image surface without intolerably scaling down or boosting the mean brightness. In the output images of GC algorithm (Fig. 7(b)) the background illumination appears to be almost uniform. However, it blurs the structures present in the dermoscopy images. The VF algorithm introduces processing-induced color artifacts as seen in Fig. 7(c). It produces output images that are unnatural in appearance. Output images of the ST in Fig.7(d) look significantly darker than the corresponding input images. The background illumination remains as uneven in the dermatological photographs. But in Fig. 7(e), an uniform background illumination is noticed throughout the image surface. Moreover, the mean brightness is not down-scaled or boosted. The structures present in the 
output images remain sharper, appear natural and does not cause any processingintroduced color artifacts. With respect to the subjective quality of the devignetted images, the proposed devignetting algorithm is superior to ST, VF and GC methods. The qualitative evaluation is repeated for hundred test images and it is found that, the proposed algorithm is consistently better than its alternatives on all test images.

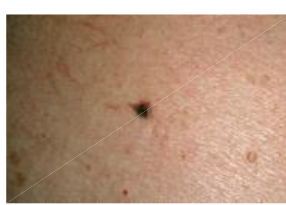

(a)

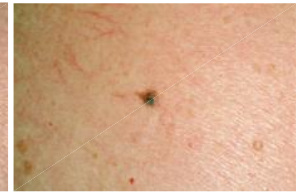

(b)

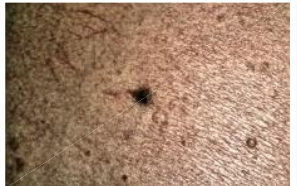

(c)

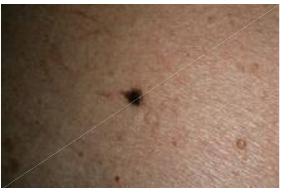

(d)

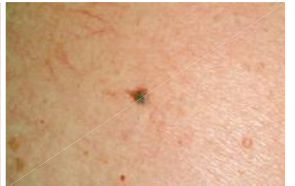

(e)

Fig. 7: Output images of different devignetting schemes for the input image (a) Input image (b) GC (c) VF (d) ST (e) Proposed

The obtained numerical values of AGIC, LOE, SFF, VSI, and computational time for different schemes ST, VF, GC and the proposed algorithm are presented in Table 2-6, respectively. As given in Table 2, the minimum value of AGIC indicates that, the background illumination in output images of the proposed method is uniform. Furthermore, in Table 3 the low values of LOE justify that the output image of the proposed algorithm is natural in appearance. In addition, Table 4 shows a highest value of SFF in the proposed algorithm indicates that, the color as well as structural distortions is negligible in the output image. Moreover, the higher value of VSI shown in Table 5 justify that, visual saliency maps of the output images are identical to that of the visual saliency maps of the corresponding input images. Therefore, the loss of salient information is negligible in the proposed algorithm is guaranteed. Finally, in Table 6 it is evident that, the proposed algorithm is computationally faster than the other methods. All these results emphasize the dominance of the proposed scheme in terms of uniformity in background illumination, information preservation and computational speed.

Table 2: AGIC scorefor different schemes in illumination-correction

\begin{tabular}{ccccc}
\hline Method & Image 1 & Image 2 & Image 3 & Summary of 100 images \\
\hline \hline ST & 0.5202 & 0.5012 & 0.5988 & $0.5401 \pm 0.0517$ \\
VF & 0.3880 & 0.3628 & 0.4278 & $0.3929 \pm 0.0328$ \\
GC & 0.1939 & 0.1560 & 0.2964 & $0.2154 \pm 0.0726$ \\
Proposed & 0.1828 & 0.1547 & 0.2310 & $\mathbf{0 . 1 8 9 5} \pm \mathbf{0 . 0 3 8 6}$ \\
\hline
\end{tabular}

Table 3: LOE score for different schemes in illumination-correction

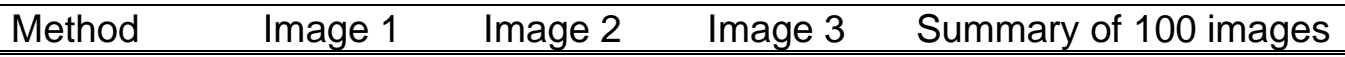




\begin{tabular}{ccccc}
\hline ST & 1383 & 1587 & 1472 & $1480 \pm 102.2758$ \\
VF & 637.4233 & 964.4730 & 753.8274 & $785.2412 \pm 165.7724$ \\
GC & 2498 & 2478 & 2476 & $2484 \pm 12.1655$ \\
Proposed & 96.1730 & 377.4392 & 225.2537 & $\mathbf{2 3 2 . 9 5 5 3} \pm \mathbf{1 4 0 . 7 9 1 2}$ \\
\hline
\end{tabular}

Table 4: SFF scorefor different schemes in illumination-correction

\begin{tabular}{ccccc}
\hline Method & Image 1 & Image 2 & Image 3 & Summary of 100 images \\
\hline ST & 0.8900 & 0.8836 & 0.8874 & $0.8870 \pm 0.0032$ \\
VF & 0.9369 & 0.9022 & 0.9249 & $0.9213 \pm 0.0176$ \\
GC & 0.8849 & 0.8693 & 0.8721 & $0.8754 \pm 0.0083$ \\
Proposed & 0.9834 & 0.9855 & 0.9661 & $\mathbf{0 . 9 7 8 3} \pm \mathbf{0 . 0 1 0 6}$ \\
\hline
\end{tabular}

Table 5: VSI scorefor different schemes in illumination-correction

\begin{tabular}{ccccc}
\hline Method & Image 1 & Image 2 & Image 3 & $\begin{array}{c}\text { Summary of 100 } \\
\text { images }\end{array}$ \\
\hline \hline ST & 0.8282 & 0.7805 & 0.8457 & $0.8181 \pm 0.0337$ \\
VF & 0.8938 & 0.8886 & 0.8815 & $0.8880 \pm 0.0062$ \\
GC & 0.8302 & 0.7782 & 0.8428 & $0.8171 \pm 0.0342$ \\
Proposed & 0.9927 & 0.9896 & 0.9886 & $\mathbf{0 . 9 9 0 3 \pm 0 . 0 0 2 1}$ \\
\hline
\end{tabular}

Table 6: Computational time for different schemes in illumination-correction

\begin{tabular}{ccccc}
\hline Method & Image 1 & Image 2 & Image 3 & $\begin{array}{c}\text { Summary of 100 } \\
\text { images }\end{array}$ \\
\hline \hline ST & 0.108442 & 0.057055 & 0.060224 & $0.0752 \pm 0.0288$ \\
VF & 108.666069 & 94.76729 & 105.5663 & $102.9999 \pm 7.2961$ \\
GC & 0.124003 & 0.096456 & 0.079563 & $0.1000 \pm 0.0224$ \\
Proposed & 2.070523 & 2.435887 & 1.575264 & $\mathbf{2 . 0 2 7 2} \pm \mathbf{0 . 4 3 1 9}$ \\
\hline
\end{tabular}

\subsection{Validation of RICE algorithm}

Contrast enhancement is done to increase the grey level difference between lesion and background. Objective evaluation is done with the help of quality metrics like SFF, VSI, PCQI and OCM. The different techniques considered for comparing the performance of contrast enhancement are, CLAHE [21], CVC [24] and LDR [25].

While evaluating the performance of the RICE algorithm a set of low contrast dermoscopy images are used. Output images produced by different contrast enhancement techniques are depicted in Fig. 8. An ideal enhancement algorithm increases the grey-scale difference without changing the mean brightness of the image. In Fig. 8(b) and 8(d), both CLAHE and LDR algorithms made a over enhancement in the image. Similarly in Fig. 8(c), multiple illumination artifacts are visible at the background region after the enhancement by 
the CVC algorithm. Besides, the proposed RICE algorithm effectively enhances the images without affecting the mean brightness of dermoscopy images is shown in Fig. 8(e). Hence, based on the subjective analysis, it is concluded that the RICE algorithm can efficiently enhance the dermoscopy image.

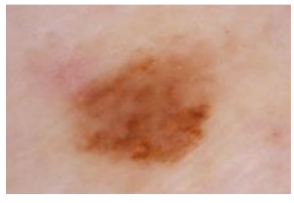

(a)

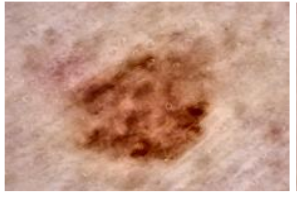

(b)

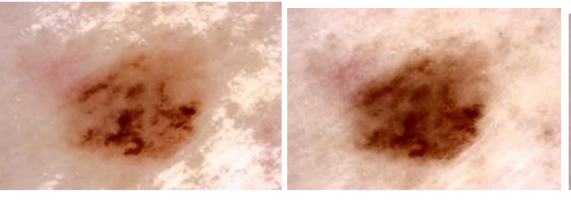

(c)

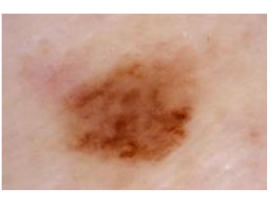

(e)

Fig. 8 Output images produced by different contrast enhancement algorithms (a) Test image (b) CLAHE (c) CVC (d) LDR (e) RICE

SFF, VSI, PCQI and OCM values for the output images produced by different schemes CLAHE, CVC, LDR and RICE are presented in Table 7-10. A higher value of SFF in RICE algorithm reflects in lesser structural distortions present in the output. Likewise, the higher value of VSI score in the proposed algorithm indicates that, the visual saliency map of the output image is identical to that of the input image. Similarly, the high values of PCQI score in RICE algorithm indicate the proper enhancement of dermoscopy images. The low value of OCM score in the proposed result indicates negligible noise amplification during enhancement. Considering the factors like enhancement in contrast, visual saliency, feature preservation and information fidelity together the RICE algorithm offers better performance compared to other algorithms.

Table 7 SFF scores for different schemes in contrast enhancement

\begin{tabular}{ccccc}
\hline Method & Image 1 & Image 2 & Image 3 & Summary of 100 images \\
\hline \hline CLAHE & 0.5943 & 0.5473 & 0.7069 & $0.6162 \pm 0.0820$ \\
CVC & 0.9362 & 0.9438 & 0.9608 & $0.9469 \pm 0.0126$ \\
LDR & 0.9786 & 0.9479 & 0.9780 & $0.9682 \pm 0.0176$ \\
RICE & 0.9965 & 0.9964 & 0.9945 & $\mathbf{0 . 9 9 5 8 \pm 0 . 0 0 1 1}$ \\
\hline
\end{tabular}

Table 8 VSI scores for different schemes in contrast enhancement

\begin{tabular}{ccccc}
\hline Method & Image 1 & Image 2 & Image 3 & Summary on 100 images \\
\hline \hline CLAHE & 0.9191 & 0.8768 & 0.9040 & $0.9000 \pm 0.0214$ \\
CVC & 0.9566 & 0.9162 & 0.9517 & $0.9415 \pm 0.0220$ \\
LDR & 0.9790 & 0.9251 & 0.9663 & $0.9568 \pm 0.0282$ \\
RICE & 0.9958 & 0.9972 & 0.9954 & $\mathbf{0 . 9 9 6 1 \pm 0 . 0 0 0 9}$ \\
\hline
\end{tabular}

Table 9 PCQI scores for different schemes in contrast enhancement 


\begin{tabular}{ccccc}
\hline Method & Image 1 & Image 2 & Image 3 & Summary on 100 images \\
\hline \hline CLAHE & 0.2680 & 0.1622 & 0.2020 & $0.2107 \pm 0.0534$ \\
CVC & 1.1603 & 1.3073 & 1.1561 & $1.2079 \pm 0.0861$ \\
LDR & 1.1445 & 1.3481 & 1.1770 & $1.2232 \pm 0.1094$ \\
RICE & 2.7586 & 2.8221 & 2.8510 & $\mathbf{2 . 8 1 0 6 \pm 1 . 6 7 3 6}$ \\
\hline
\end{tabular}

Table 10 OCM scores for different schemes in contrast enhancement

\begin{tabular}{ccccc}
\hline Method & Image 1 & Image 2 & Image 3 & Summary on 100 images \\
\hline \hline CLAHE & 0.5017 & 0.1164 & 0.6459 & $0.4213 \pm 0.2737$ \\
CVC & 0.4507 & 0.0142 & 0.0301 & $0.1650 \pm 0.2476$ \\
LDR & 0.2295 & 0.0451 & 0.0481 & $0.1076 \pm 0.1056$ \\
RICE & 0.0515 & 0.0102 & 0.0513 & $\mathbf{0 . 0 3 7 7 \pm 0 . 0 2 3 8}$ \\
\hline
\end{tabular}

\subsection{Validation of Unsharp Masking}

The quality of the sharpened image is influenced by the parameter $\lambda$ in Unsharp Masking. This process is carried out by varying the value of $\lambda$ from 0 to 5 with an interval of 0.5. It is analyzed subjectively using BRISQUE and CPBD.

The sharpening effect gets increased when the value of $\lambda$ increases and it can be clearly observed from the images depicted in Fig. 9(b) $-9(f)$. When the value of $\lambda$ is less than 1, the sharpening effect is less as illustrated in Fig. 9(e) - Fig. 9(f). In Fig. 9(b) -9(c), it is observed that the value of $\lambda$ increases beyond 2.5 , the non-edge fine texture gets amplified which may adversely affect the segmentation process. Hence, based on the perceived quality of the processed images, the range of the $\lambda$ between 1.5 and 2.5 is observed to be ideal for Unsharp Masking in dermoscopy images.

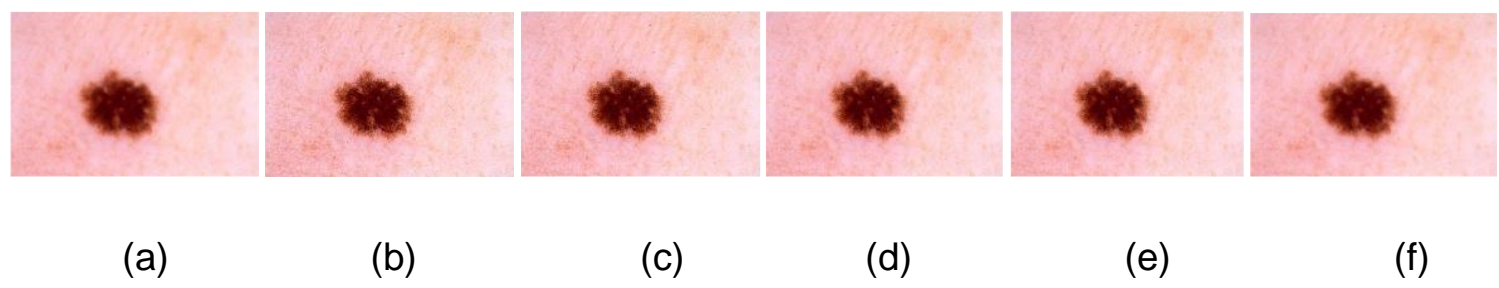

Fig. 9: Output images produced by Unsharp Masking for different values of $\lambda(a)$ Input image

$$
\text { (b) } \lambda=5 \text { (c) } \lambda=2.5 \text { (d) } \lambda=1 \text { (e) } \lambda=0.5 \text { (f) } \lambda=0
$$

The variations of BRISQUE and CPBD metrics to different values of $\lambda$ are shown in Fig. 10. BRISQUE exhibits an inverted bell-shaped curve for three test images. BRISQUE shows, low values when the range of the $\lambda$ is between 1.5 and 2 . The value of the CPBD metric increases as the $\lambda$ increases from 0.5 to 5 . The slope of the CPBD starts decreasing 
when the value of the $\lambda$ is greater than 2. The variations of BRISQUE and CPBD to $\lambda$ indicate that the optimum range of $\lambda$ is between 1.5 and 2.5 .

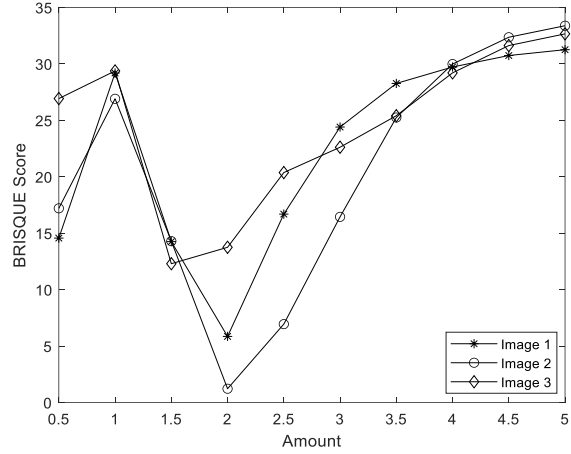

(a)

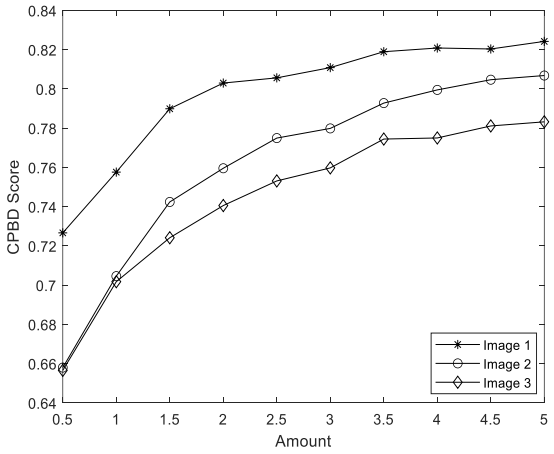

(b)

Fig. 10: Variation of BRISQUE and CPBD score for different values of the $\lambda$

(a) BRISQUE Vs $\lambda$ (b) CPBD Vs $\lambda$

The Unsharp Masking algorithm is compared both qualitatively and quantitatively against the Local Laplacian filter [15]. Output images for different sharpening algorithms are shown in Fig. 11. From the output of the Local Laplacian filter in Fig. 11(b), it is evident that this filter excessively sharpens the images, which results in amplification of non-edge fine texture. On the other hand in Fig. 11(c), an ideal sharpening algorithm is able to strengthen the lesion without amplifying the non-edge fine texture in the image.

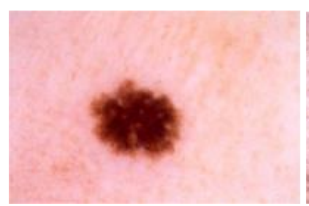

(a)

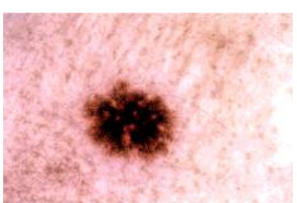

(b)

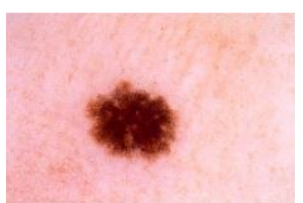

(c)

Fig. 11 Input image and results produced by sharpening filters (a) input image (b) output of Local laplacian filter (c) Output of Unsharp Masking

The values of BRISQUE scores for the output produced by Unsharp Masking and Local Laplacian filter for 100 images are presented in Table 11. It can be observed that, Unsharp Masking exhibits the lowest values of BRISQUE compared to the Local Laplacian filter. Low values of the BRISQUE score shows that the image has sharpened without the amplification of non-edge fine textures.

Table 11: BRISQUE score obtained for Unsharp Masking and Local Laplacian filter

\begin{tabular}{ccccc}
\hline Method & Image 1 & Image 2 & Image 3 & $\begin{array}{c}\text { Summary of 100 } \\
\text { images }\end{array}$ \\
\hline \hline
\end{tabular}




$\begin{array}{crrrr}\text { Local laplacian filter } & 22.7326 & 26.9124 & 31.5786 & 27.0745 \pm 4.4252 \\ \text { Unsharp masking } & 5.8528 & 1.2185 & 13.7466 & \mathbf{6 . 9 3 9 3 \pm} \mathbf{6 . 3 3 4 3}\end{array}$

\subsection{Validation of reflection removal}

The selection of the Smoothing Parameter (SP) and Rate Control Parameter (RCP) of the reflection removed images are analyzed subjectively in this section. SP controls the degree of smoothening and RCP determines the number of iterations. The small value of $\mathrm{RCP}$ needs more iterations and results in sharper output image. For this analysis, a range of suitable dermatological photographs are identified that possess specular reflection. The outputs of the reflection removal algorithm, corresponding to the test image are depicted in Fig. 12 - 14.

The value of SP is varied between 0.01 to 0.04 and for each value of SP, RCP is varied between 1.1 to 2 with an interval of 0.1 . It is apparent from the output images that, as the value of SP increase beyond 0.02 , unexpectedly the image gets smoothed heavily with cartoon artifact. Moreover, as the value of SP increases the data loss occurs which can be inferred from Fig. 14. When the value of SP is less than 0.02 , the reflected part of the image is also removed without the smoothing effect as presented in Fig. 13. When SP is 0.01 , it is observed that reflection is not properly removed from the image as shown in Fig. 12. Based on the perceived quality of processed images, the ideal value of SP is 0.02 for dermoscopy images. From Fig. 12(e), Fig. 13(e) and Fig. 14(e) it can be observed that as the value of $\mathrm{RCP}$ is less than 1.5, the information contained in the image is lost with visible cartoon artifact. When the value of RCP increases above 1.8, reflection from dermoscopy images is not efficiently removed as shown in Fig. 12(b),Fig. 13(b) and Fig. 14(b). Thus, based on the perceived quality of the resulting images, the range of RCP between 1.5 and 1.7 is observed to be ideal for the dermoscopy images.

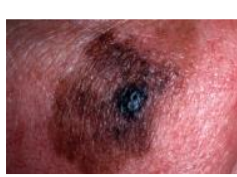

(a)

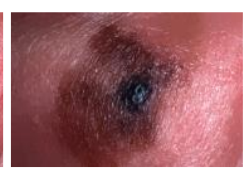

(b)

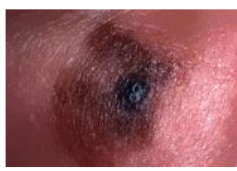

(c)

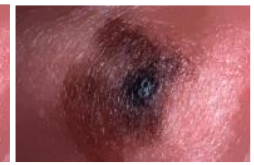

(d)

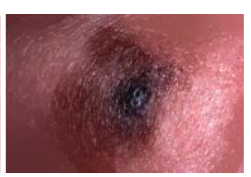

(e)

Fig. 12: Output images of reflection removal for various values of $\mathrm{RCP}(\mathrm{SP}=0.01)$ (a) Input Image (b) $R C P=1.9$ (c) $R C P=1.7$ (d) $R C P=1.5$ (e) $R C P=1.3$ 


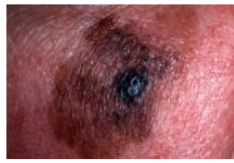

(a)

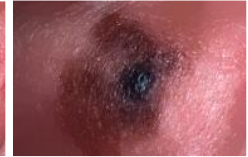

(b)

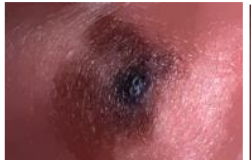

(c)

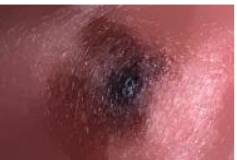

(d)

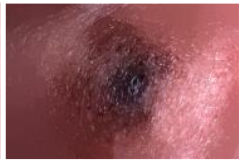

(e)

Fig. 13 Output images of reflection removal for various values of $\mathrm{RCP}(\mathrm{SP}=0.02)$ (a) Input Image (b) $R C P=1.9$ (c) $R C P=1.7$ (d) $R C P=1.5$ (e) $R C P=1.3$

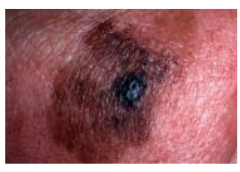

(a)

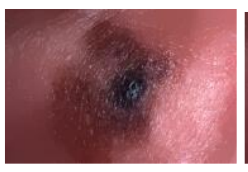

(b)

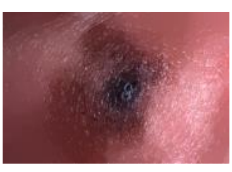

(c)

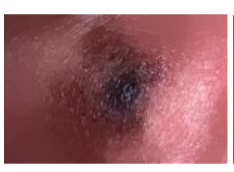

(d)

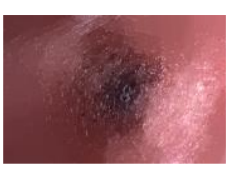

(e)

Fig. 14 Output images of reflection removal for various values of $\mathrm{RCP}(\mathrm{SP}=0.03)$ (a) Input image (b) $R C P=1.9$ (c) $R C P=1.7$ (d) $R C P=1.5$ (e) $R C P=1.3$

\subsection{Validation of phase congruency based virtual shaving}

The influence of the threshold on the subjective quality of the virtually-shaved images is analyzed subjectively as well as objectively. The quality assessment is done objectively using Peak Signal to Noise Ratio (PSNR) and Structural Similarity Index Metric (SSIM). The test image and its Ground-Truth (GT) image used for virtual shaving are shown in Fig. 15. The value of the threshold is varied from 0.55 to 1 . When the value of the threshold is between 0.55 and 0.7 , almost no hairs are removed from the dermoscopy image as depicted in Fig. 16(a). But hairs are completely removed in Fig. 16(b) when the threshold value is increased beyond 0.85 . However, if the value of the threshold is increased above 0.95 , the image information content is also lost along with the removed hair is shown in Fig. 16(c). Hence, based on the perceived quality of the processed images, the range of threshold between 0.85 and 0.9 is observed to be ideal for dermatological photographs.

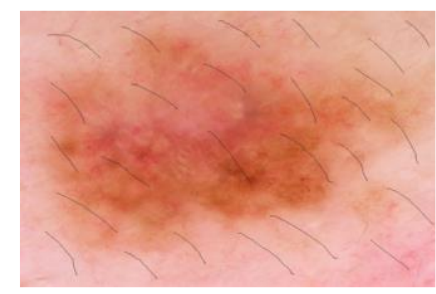

(a)

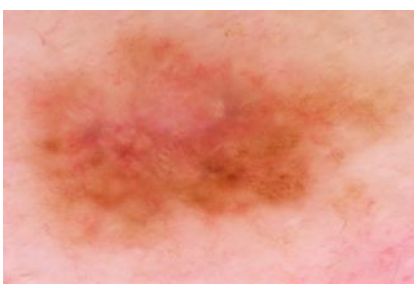

(b) 
Fig. 15 Representative test image containing hairs (a) Image (b) GT

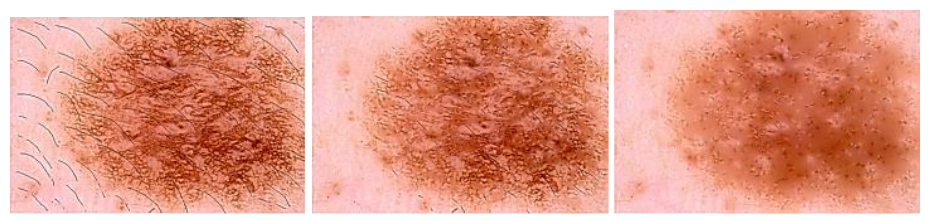

(a)

(b)

(c)

Fig. 16 Outputs images of the phase congruency-based virtual shaving (a) threshold $=0.7$

(b) threshold $=0.85(\mathrm{c})$ threshold $=0.95$

The variations of PSNR and SSIM to various values of the threshold are shown in Fig. 17. PSNR and SSIM metrics are computed between the virtually-shaved image and the ground-truth image. Both PSNR and SSIM remain consistent for threshold values less than 0.6. But, when threshold increases beyond 0.6 , both the parameters exhibit a bell-shaped curve. PSNR has its maximum value when the threshold is between 0.75 and 0.85 and SSIM reaches its maximum values when the threshold is between 0.75 and 0.9 . A higher value of PSNR and SSIM justifies that, the output of the virtually-shaved image and groundtruth image are identical. Hence, it is concluded that, from the variations of PSNR and SSIM the optimum range of threshold for virtual shaving of dermoscopy images is between 0.75 and 0.9 .

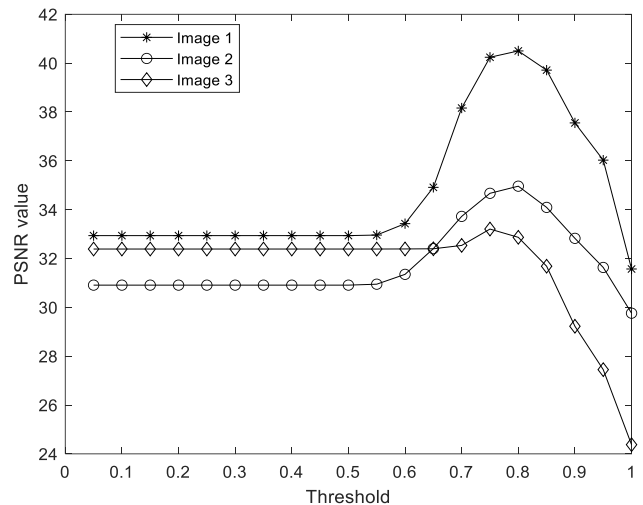

(a)

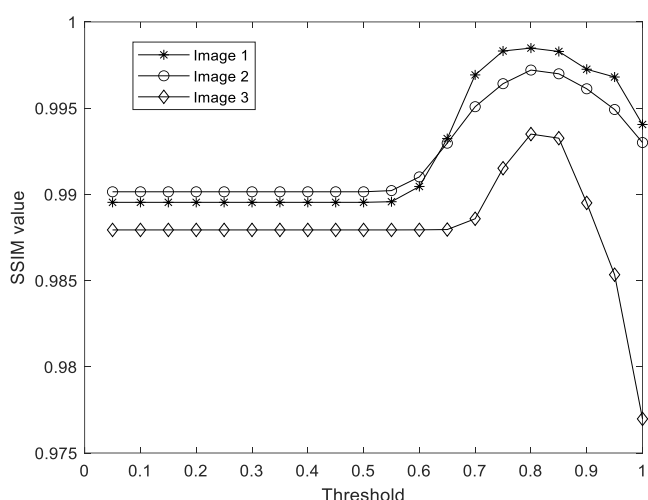

(b)

Fig. 17: PSNR and SSIM plotted for different values of threshold (a) PSNR versus threshold (b) SSIM versus threshold

\subsection{Validation of RELM based Segmentation}

In this section, different segmentation algorithms are applied to the preprocessed and without pre-processed dermoscopy images. The performance of different algorithms is 
compared subjectively as well as objectively. The quality metrics like DSI, JI and TSC [27] are used for objective comparison. The different segmentation algorithm used are FCM [28], Isolate Thresholding method (IT) [29], k-means [30], and RELM.

The output of different segmentation algorithms without pre-processing is shown in Figure 18. Here, the skin lesion are not segmented accurately because of the existence of noise, non-uniform illumination and hairs. The virtually-shaved image with a threshold value of 0.85 along with the manually segmented ground-truth and output of different segmentation algorithms are depicted in Figure 19. From the output results of FCM, IT and k-means (Figure 19(c) - 19(e)) the algorithms failed to segment the skin lesions properly. The output of RELM agrees with the manual segmentation and effectively segments the skin lesions (Figure 19 (f)). Thus, based on subjective quality, it can be concluded that the RELM algorithm is able to segment skin lesions efficiently from the dermoscopy images.

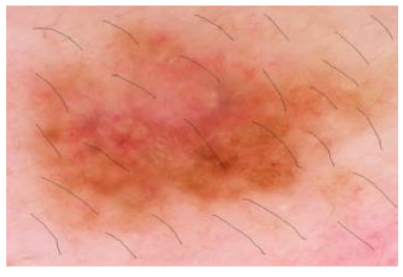

(a)

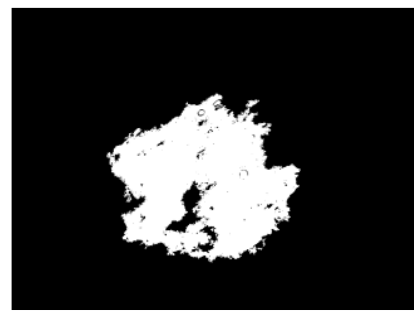

(d)

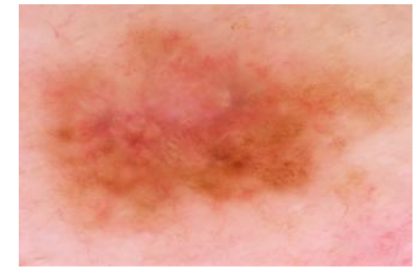

(b)

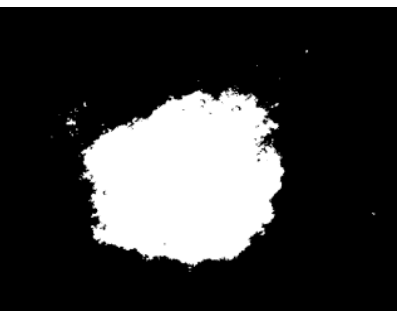

(e)

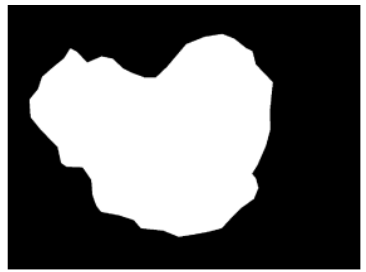

(c)

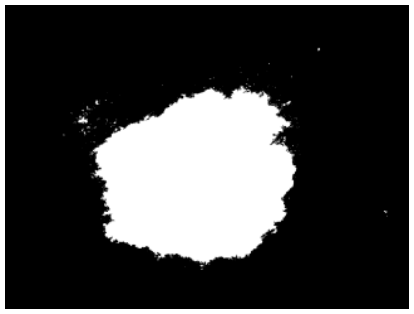

(f)

Figure 18: Output images of different segmentation algorithms without pre-processing (a) Raw image (b) ground truth image (c) FCM (d) IT (e) k-means (f) RELM 


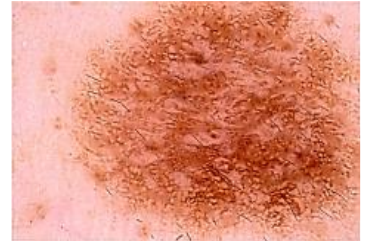

(a)

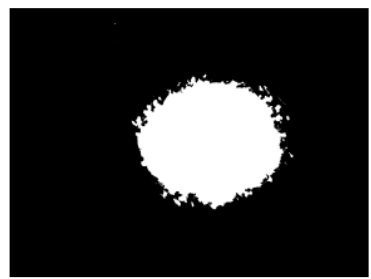

(d)

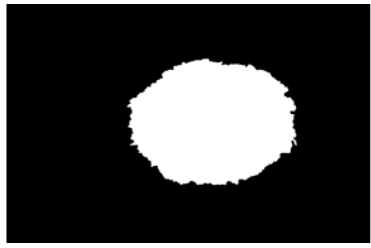

(b)

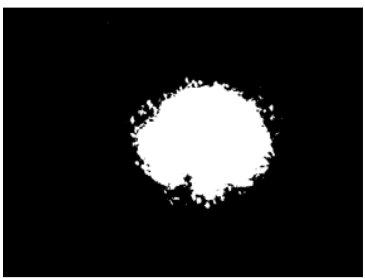

(e)

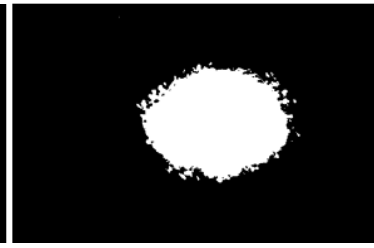

(c)

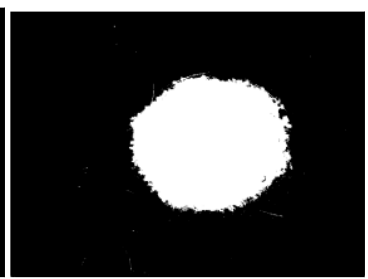

(f)

Figure 19: Output images of different segmentation algorithms for the Pre-processed image (a) virtually shaved image (b) ground truth image (c) FCM (d) IT (e) k-means (f) RELM

The values of JI, DSI and TSC calculated for 100 dermoscopy images for different segmentation algorithms with pre-processing and without pre-processing are tabulated in Table 12 - 14 respectively. The skin lesions segmented manually by experts are used as the ground-truth for the calculation of different quality metrics. A high value of JI, DSI and TSC indicate that the segmented lesions agree with ground-truth. RELM algorithm exhibits the highest value for all three metrics. This indicates that the RELM algorithm has produced more accurate segmentation results compared to other schemes. Objective evaluation results agree with the inferences drawn by the subjective evaluation for the similarity of the skin lesions segmented using different algorithms with ground truth.

Table 12: Jl score shown by different segmentation schemes

\begin{tabular}{|c|c|c|c|c|c|c|c|}
\hline Method & $\begin{array}{c}\text { Image } \\
\mathbf{1}\end{array}$ & $\begin{array}{c}\text { Image } \\
\mathbf{2}\end{array}$ & $\begin{array}{c}\text { Image } \\
\mathbf{3}\end{array}$ & $\begin{array}{c}\text { Image } \\
\mathbf{4}\end{array}$ & $\begin{array}{c}\text { Image } \\
\mathbf{5}\end{array}$ & $\begin{array}{c}\text { Summary of } \\
\mathbf{1 0 0} \text { images } \\
\text { (Without pre- } \\
\text { processing) }\end{array}$ & $\begin{array}{c}\text { Summary of } \\
\mathbf{1 0 0} \text { images } \\
\text { (With pre- } \\
\text { processing) }\end{array}$ \\
\hline FCM & 0.8313 & 0.7666 & 0.5907 & 0.7764 & 0.8928 & $0.7062 \pm 0.1024$ & $0.7295 \pm 0.1245$ \\
\hline IT & 0.8584 & 0.7726 & 0.5964 & 0.7870 & 0.8931 & $0.7112 \pm 0.1126$ & $0.7425 \pm 0.1336$ \\
\hline $\begin{array}{c}\text { k- } \\
\text { means }\end{array}$ & 0.7787 & 0.6502 & 0.0085 & 0.7388 & 0.8555 & $0.4372 \pm 0.3878$ & $0.4791 \pm 0.4126$ \\
\hline RELM & 0.8729 & 0.9504 & 0.6972 & 0.8771 & 0.9708 & $\mathbf{0 . 8 9 7 1 \pm 0 . 0 1 3 4}$ & $\mathbf{0 . 9 4 0 2 \pm 0 . 1 2 9 7}$ \\
\hline
\end{tabular}


Table 13: DSI score shown by different segmentation schemes

\begin{tabular}{|c|c|c|c|c|c|c|c|}
\hline Method & $\begin{array}{c}\text { Image } \\
1\end{array}$ & $\begin{array}{c}\text { Image } \\
2\end{array}$ & $\begin{array}{c}\text { Image } \\
3\end{array}$ & $\underset{4}{\text { Image }}$ & $\begin{array}{c}\text { Image } \\
5\end{array}$ & $\begin{array}{l}\text { Summary of } \\
100 \text { images } \\
\text { (Without pre- } \\
\text { processing) }\end{array}$ & $\begin{array}{c}\text { Summary of } \\
100 \text { images } \\
\text { (With pre- } \\
\text { processing) }\end{array}$ \\
\hline FCM & 0.9079 & 0.8679 & 0.7427 & 0.8741 & 0.9434 & $0.7865 \pm 0.0456$ & $0.8395 \pm 0.0862$ \\
\hline $\mathrm{IT}$ & 0.9238 & 0.8717 & 0.7472 & 0.8808 & 0.9435 & $0.7989 \pm 0.0812$ & $0.8476 \pm 0.0907$ \\
\hline $\begin{array}{c}\mathrm{k}- \\
\text { means }\end{array}$ & 0.8756 & 0.7880 & 0.0169 & 0.8498 & 0.9221 & $0.4961 \pm 0.3214$ & $0.5602 \pm 0.4725$ \\
\hline RELM & 0.9322 & 0.9746 & 0.8216 & 0.9345 & 0.9852 & $0.8545 \pm 0.0462$ & $0.9895 \pm 0.0790$ \\
\hline
\end{tabular}

Table 14: TSC score shown by different segmentation schemes

\begin{tabular}{|c|c|c|c|c|c|c|c|}
\hline Method & $\begin{array}{c}\text { Image } \\
\mathbf{1}\end{array}$ & $\begin{array}{c}\text { Image } \\
\mathbf{2}\end{array}$ & $\begin{array}{c}\text { Image } \\
\mathbf{3}\end{array}$ & $\begin{array}{c}\text { Image } \\
\mathbf{4}\end{array}$ & $\begin{array}{c}\text { Image } \\
\mathbf{5}\end{array}$ & $\begin{array}{c}\text { Summary of } \\
\text { 100 images } \\
\text { (Without pre- } \\
\text { processing) }\end{array}$ & $\begin{array}{c}\text { Summary of } \\
\text { 100 images } \\
\text { (With pre- } \\
\text { processing) }\end{array}$ \\
\hline FCM & 0.8310 & 0.7664 & 0.5909 & 0.7763 & 0.8924 & $0.7012 \pm 0.1156$ & $0.7294 \pm 0.1242$ \\
\hline IT & 0.8581 & 0.7725 & 0.5965 & 0.7869 & 0.8927 & $0.7202 \pm 0.1004$ & $0.7424 \pm 0.1334$ \\
\hline $\begin{array}{c}\text { k- } \\
\text { means }\end{array}$ & 0.7784 & 0.6500 & 0.0085 & 0.7387 & 0.8551 & $0.4241 \pm 0.3941$ & $0.4790 \pm 0.4125$ \\
\hline RELM & 0.9984 & 0.9536 & 0.7026 & 0.8857 & 0.9881 & $\mathbf{0 . 9 3 2 8 \pm 0 . 1 3 6 1}$ & $\mathbf{0 . 9 5 4 9 \pm 0 . 1 5 9 4}$ \\
\hline
\end{tabular}

RELM algorithm is used to segment the lesions from the preprocessed images. It exhibits a JI, DIS and TSC score higher than FCM, IT and k-means, shows that automated segmentation of RELM algorithm are more accurate with the manual segmentation of skin lesions in dermoscopy images.

\section{CONCLUSION}

In this paper, different enhancement techniques are introduced for preprocessing of dermoscopy images. Here, the optimization based framework is tested with data archive of ISIC(2016). Based on the results obtained with and without pre-processed segmentation, it is concluded that the implementation of pre-processing algorithm improves the success rate in RGB images. The NLM filter have been found to preserve very fine details by removing the noise in skin lesion images. Also, the NLM filter exhibits the lowest BRISQUE score compared to anisotropic diffusion filter and bilateral filter. The proposed RICE algorithm for contrast enhancement method is found to be superior to the existing methods including CLAHE, LDR and CVC with better SFF, VSI, PCQI and OCM scores. The enhancement of dermoscopy images is further improved by eliminating the undesired information due to 
reflection using reflection removal method. Also, in our framework virtual shaving is included to remove the hairs without any loss of image content with appreciably high PSNR and SSIM metrics. The values of quality evaluation metrics like PSNR and SSIM are appreciably high for output images produced by phase congruency-based virtual shaving when the value of the threshold is in the range of $0.85-0.9$. However, the proposed system generates better results among all comparable methods in terms of qualitative and quantitative aspects. Therefore, the introduced pre-processing framework is more appropriate for low quality melanoma images. From the score of quality metrics like Disc Similarity Index, Jacquard Index and Total Segmentation Coefficient, it has been concluded that when pre-processing steps are used in the proper sequence, segmentation using Regularized Extreme Learning Machine is more efficient than other algorithms.

\section{ABBREVATIONS:}

NLM - Non-Local Means

RICE - Robust Image Contrast Enhancement

DSI - Disc Similarity Index

$\mathrm{JI}$ - Jacquard Index

TSC - Total Segmentation Coefficient

RELM - Regularized Extreme Learning Machine

AWB - Automatic White Balance

CLAHE - Contrast Limited Adaptive Histogram Equalization

CVC - Contextual and Variational Contrast enhancement algorithm

LDR - Layered Difference Representation

DoS - Degree of Smoothing

SD - Standard Deviation

DQP - Devignetting Quality Parameter

BRISQUE - Blind Reference less Image Spatial Quality Evaluator

AGIC - Average Gradient of the Illumination Component

LOE - Lightness Order Error 
SFF - Sparse Feature Fidelity

VSI - Visual Saliency-based Index

PCQI - Patch-based Contrast Quality Index

OCM - Over-Contrast measure

CPBD - Cumulative Probability of Blur Detection

SP - Smoothing Parameter

RCP - Rate Control Parameter

PSNR - Peak Signal to Noise Ratio

SSIM - Structural Similarity Index Metric

\section{DECLARATION:}

Availability of data and material:

Not applicable

\section{Competing Interests:}

Author A has no conflict of Interest

Author B has no conflict of Interest

Author $\mathrm{C}$ has no conflict of Interest

\section{Funding:}

This study has no funding

\section{Author's Contributions:}

Jeba Derwin : Conceptualization, Methodology, Writing- Reviewing and Editing.

Jeba Singh : Writing- Original draft preparation.

Priestly Shan : Visualization, Investigation, Supervision.

\section{Acknowledgements:}

Not applicable

\section{Authors's information:}

D. Jeba Derwin received her B.E degree in Electronics and Communication

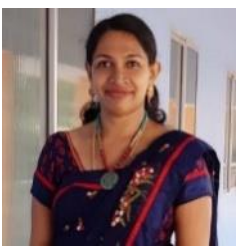
Engineering from Anna University in 2005 and M.E degree in Communication systems from Anna University in 2007. She received her 
Ph.D degree from Anna University, Chennai in 2020. Currently, she is working as Assistant Professor in the department of Electronics and Communication Engineering of SRM TRP Engineering college. Her current research interest includes Biomedical Image processing, pattern Recognition and Deep learning.

B. Priestly Shan received his B.E degree in Electronics and Communication Engineering from MS university in 2003 and Masters in Communication systems from Anna University in 2006. He received his Ph.D degree from Anna University of Technology, Coimbatore in 2011. Currently, he is working as Dean Academic Affairs at Chandiargh University, Punjab. His current research interest includes Biomedical Image processing, pattern Recognition and Deep learning.

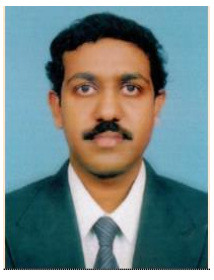

O. Jeba Singh received his BE degree in Electrical and Electronics from Manonmaniam Sundaranar University in 2001 and his M.E degree in power systems from Annamalai University in 2004. He received his Ph.D degree from Anna University Chennai in 2019. Currently, he is working as Associate Professor in the department of Electrical and Electronics Engineering of Arunachala college of Engineering for women. His current research interest includes power quality, PV systems and Image processing.

\section{REFERENCES}

[1] R. L. Siegel, K. D. Miller, and A. Jemal, "Cancer statistics, 2018," CA: A Cancer Journal of Clinicians, vol. 68(1), pp. 7-30, 2018.

[2] F. Bray, J. Ferlay, I. Soerjomataram, R. L. Siegel, L. A. Torre, and A. Jemal, "Global cancer statistics 2018: GLOBOCAN estimates of incidence and mortality worldwide for 36 cancers in 185 countries," CA: A Cancer Journal of Clinicians, vol. 68(6), pp. 394-424, 2018.

[3] M. L. Bafounta, A. Beauchet, P. Aegerter, and P. Saiag, "Is dermoscopy (epiluminescence microscopy) useful for the diagnosis of melanoma? Results of a metaanalysis using techniques adapted to the evaluation of diagnostic tests," Archives of Dermatology, vol. 137(10), pp. 1343-1350, 2001.

[4] K. Madhankumar and P. Kumar, "Characterization of skin lesions," International Conference on Pattern Recognition, Informatics and Medical Engineering (PRIME-2012), pp. 302-306, 2012.

[5] Jaworek-Korjakowska $J$ "Novel method for border irregularity assessment in dermoscopic color images" Computational and Mathematical Methods in Medicine,Vol.2015, pp.1-11,2015.

[6] Restrepo Parra E., Ocampo Blandón C. F., Riaño Rojas J. C., and Jaramillo Ayerbe P. F., "Contrast enhancement by searching discriminant color projections in dermoscopy images”, Revista Facultad Ingenieria ,University Antioquia, Vol. 79, pp. 192-200, 2016. 
[7] N. K. Mishra and M. E. Celebi, "An Overview of Melanoma Detection in Dermoscopy Images Using Image Processing and Machine Learning", arxiv.org: 1601.07843,2016.

[8] O. Cherepkova \& J. Y. Hardeberg, "Enhancing dermoscopy images to improve melanoma detection," 2018 Colour and Visual Computing Symposium (CVCS), pp. 1-6, 2018.

[9] Jayalakshmi D.,\& Dheeba J.,"Border Detection in Skin Lesion Images Using an Improved Clustering Algorithm", International Journal of e-Collaboration (IJeC), Vol.16(4), pp.1529,2020 .

[10] Nadia Smaoui Zghal\& Nabil Derbel, "Melanoma Skin Cancer Detection based on Image Processing", Current Medical Imaging,Vol.16(1), 2020.

[11] Pankaj Kandhway, Ashish Kumar Bhandari, Anurag Singh, "A novel reformed histogram equalization based medical imagecontrast enhancement using krill herd optimization," Biomedical Signal Processing and Control, vol. 56,2020.

[12]S. Jeevakala , A. Brintha Therese, "Sharpening enhancement technique for MR images to enhance thesegmentation," Biomedical Signal Processing and Control, vol. 41 pp. 2130,2018 .

[13]Yeong-Cheol Heo , Kyuseok Kim , Youngjin Lee ," Image De-noising Using Non-Local Means (NLM) Approach in Magnetic Resonance (MR) Imaging: A Systematic Review",Applied science,Vol.10(7028), pp.1-16, 2020

[14] Duan X., "A Multiscale Contrast Enhancement for Mammogram Using Dynamic Unsharp Masking in Laplacian Pyramid", IEEE Transactions on Radiation and Plasma Medical Sciences, vol. 3, No. 5, pp. 557-564,2019.

[15] K. Gu, G. Zhai, X. Yang, W. Zhang and C. W. Chen, "Automatic Contrast Enhancement Technology With Saliency Preservation," in IEEE Transactions on Circuits and Systems for Video Technology, vol. 25, no. 9, pp. 1480-1494, 2015.

[16] Rajchel, M., Oszust, M. "No-reference image quality assessment of authentically distorted images with global and local statistics" Signal, Image and Video processing.Vol. 15, pp.83-91, 2021.

[17] Arvanitopoulos N., Achanta R., Süsstrunk S. , "Single Image Reflection Suppression", 2017 IEEE Conference on Computer Vision and Pattern Recognition (CVPR), Honolulu, HI, pp. 1752-1760,2017.

[18] J. Li, Q. Hu and M. Ai, "RIFT: Multi-Modal Image Matching Based on Radiation-Variation Insensitive Feature Transform," in IEEE Transactions on Image Processing, vol. 29, pp. 3296-3310, 2020.

[19] Gutman D., Codella N. C. F., Celebi E., Helba B., Marchetti M., Mishra N., Halpern A., "Skin lesion analysis toward melanoma detection: A challenge at the 2017 International 
symposium on biomedical imaging (ISBI), hosted by the international skin imaging collaboration (ISIC)," IEEE 15th International Symposium on Biomedical Imaging, Washington, DC, pp. 168-172,2018.

[20] Mishra D., Chaudhury S., Sarkar M., Soin A. S., Sharma V. , "Edge Probability and Pixel Relativity-Based Speckle Reducing Anisotropic Diffusion", IEEE Transactions on Image Processing, Vol. 27(2), pp. 649-664, 2018.

[21] Gavaskar R. G., Chaudhury K. N. (2019), "Fast Adaptive Bilateral Filtering”, IEEE Transactions on Image Processing, Vol. 28(2), pp. 779-790.

[22] Zhou M., Jin K., Wang S., Ye J., Qian D. (2018), "Color Retinal Image Enhancement Based on Luminosity and Contrast Adjustment", IEEE Transactions on Biomedical Engineering, Vol. 65(3), pp. 521-527.

[23] Shamsudeen F.M., Raju G., "An objective function based technique for devignetting fundus imagery using MST", Informatics in Medicine Unlocked, Vol. 14, pp. 82-91, 2019.

[24] Srinivas K., Bhandari A. K., "Low light image enhancement with adaptive sigmoid transfer function”, IET Image Processing, Vol. 14(4), pp. 668-678, 2020.

[25] Celik T., Tjahjadi T., "Contextual and Variational Contrast Enhancement", IEEE Transactions on Image Processing, Vol. 20(12), pp. 3431-3441, 2011.

[26] Lee C., Lee C., Kim C., "Contrast Enhancement Based on Layered Difference Representation of 2D Histograms", IEEE Transactions on Image Processing, Vol. 22(12), pp. 5372-5384, 2013.

[27] Liu. J, "A Cascaded Deep Convolutional Neural Network for Joint Segmentation and Genotype Prediction of Brainstem Gliomas", IEEE Transactions on Biomedical Engineering, Vol.65, No.9, pp.1943-1952, 2018.

[28] Bai. X., et. al., "Intuitionistic Center-Free FCM Clustering for MR Brain Image Segmentation", IEEE Journal of Biomedical and Health Informatics, Vol.23, No.5, pp.20392051, 2019.

[29] Quan. R., et. al., "A Novel IGBT Health Evaluation Method Based on Multi-Label Classification", IEEE Access, Vol.7, pp. 47294-47302, 2019.

[30] Jaisakthi. S.M., et. al., "Automated Skin Lesion Segmentation of Dermoscopic Images using GrabCut and K-Means Algorithms", IET Computer Vision, Vol.12, No.8, pp.1088-1095, 2018. 

